\title{
OSCILLATION AND A CLASS OF LINEAR DELAY DIFFERENTIAL EQUATIONS
}

\author{
BY
}

\author{
DAVID LOWELL LOVELADY
}

\begin{abstract}
The differential equation $u^{(m)}(t)+p(t) u(g(t))=0$, where $p$ is one-signed, is broken into four cases, according to the parity of $m$ and the sign of $p$. In each case, an analysis is given of the effect $g$ can have on oscillation properties, and oscillation and nonoscillation criteria are given.
\end{abstract}

I. Introduction. Let $q$ be a continuous function from $[0, \infty)$ to $(0, \infty)$, let $n$ be a positive integer, and let $G$ be the set to which $g$ belongs if and only if $g$ is an unbounded nondecreasing continuous function from $[0, \infty)$ to $[0, \infty)$ such that $g(t)<t$ whenever $t \geqslant 0$. We propose to study herein the four linear delay differential equations

$$
\begin{aligned}
u^{(2 n)}(t)+q(t) u(g(t)) & =0, \\
u^{(2 n+1)}(t)-q(t) u(g(t)) & =0, \\
u^{(2 n)}(t)-q(t) u(g(t)) & =0,
\end{aligned}
$$

and

$$
u^{(2 n+1)}(t)+q(t) u(g(t))=0,
$$

where $g$ is in $G$. Of particular interest are the oscillation properties of (1), (2), (3) and (4), and a comparison of these properties with the oscillation properties of

$$
\begin{aligned}
u^{(2 n)}(t)+q(t) u(t) & =0 \\
u^{(2 n+1)}(t)-q(t) u(t) & =0 \\
u^{(2 n)}(t)-q(t) u(t) & =0
\end{aligned}
$$

and

Received by the editors March 3, 1975 and, in revised form, November 25, 1975.

AMS (MOS) subject classifications (1970). Primary 34C10, 34K25; Secondary 34K15.

Key words and phrases. Delay differential equations, higher order oscillation.

- American Mathematical Society 1977 


$$
u^{(2 n+1)}(t)+q(t) u(t)=0 .
$$

By a solution of (1) we shall mean a continuous real-valued funct on $u$ on $[0, \infty)$ for which there is $b_{u} \geqslant 0$ such that $u^{(2 n)}$ exists on $\left(b_{u}, \infty\right)$ and such that (1) is true whenever $t>b_{u}$. Solutions of (2), (3) and (4) are defined analogously. A continuous real-valued function $u$ on $[0, \infty)$ is called oscillatory if and only if the set $\{t: u(t)=0\}$ is unbounded. An alternative definition of oscillatory would be that each of $\{t: u(t)>0\}$ and $\{t: u(t)<0\}$ is unhounded. For solutions of (1), (2), (3) and (4) which are not eventually trivial, these two notions of oscillation will turn out to be the same.

The relationship of (1) and (2) to (5) and (6), respectively, is that a large delay (small $g$ ) can des roy oscillation, but no delay can induce oscillation. This is an extension of second order results of T. Burton and R. Grimmer [2], P. Waltman [20], and the present author [13]. The relations'.ip of (3) and (4) to (7) and (8) is more involved. In these cases a larie delay can testroy oscillation in unbounded solutions but induce oscillatic: in bound i $i$ solutions. There is a "middle ground" in which the delay is small enough to preserve oscillation in unbounded solutions but large enough to induce oscillation in bounded solutions. In particular, we shall give conditions which ensure that every solution of (4) is oscillatory. Our conditions will be different from those given by G. Ladas, V. Lakshmikantham, and J. S. Papadakis [8, Corollary 3.2]. Our primary sources for the oscillation properties of (5), (6), (7) and (8) are [9], [10], [11] and [12], and our primary sources for delay-induced oscillation are [8], [15], [16], [17] and [18].

II. General considerations. Before studying oscillation and nonoscillation criteria for (1), (2), (3) and (4), we need information on the structure of solutions. If $u$ is a solution of (1), (2), (3) or (4), we shall call $u$ trivial if and only if $u(t)=0$ whenever $t \geqslant b_{u}$, and we shall call $u$ eventually trivial if and only if there is $c \geqslant b_{u}$ such that $u(t)=0$ whenever $t \geqslant c$.

TheOREM 1. Suppose $g$ is in $G$, and $u$ is an eventually trivial but not trivial solution of (1), (2), (3) or (4). Let $c$ be the least member of $\left[b_{u}, \infty\right)$ such that $u(t)=0$ whenever $t \geqslant c$. Then $g(c)=c$.

Corollary 1. Suppose $g$ is in $G$ and $g(t)<t$ whenever $t>0$. Then no nontrivial solution of (1), (2), (3) or (4) is eventually trivial.

THEOREM 2. Suppose $g$ is an increasing member of $G$ and $g(t)<t$ whenever $t>0$. Suppose $u$ is a solution of (1), (2), (3) or (4), and suppose $u$ has no zeros in $\left[g\left(b_{u}\right), b_{u}\right]$. Then there is no interval $[c, d]$ with $b_{u} \leqslant c<d$ such that $u(t)=0$ whenever $c \leqslant t \leqslant d$. 
THEOREM 3. Suppose $g$ is in $G$, and $u$ is an oscillatory solution of (1), (2), (3) or (4); $u$ not eventually trivial. Then each of $\{t: u(t)>0\}$ and $\{t: u(t)<0\}$ is unbounded.

Theorem 1, Corollary 1 and Theorem 2 say that some of the peculiarities of delay differential equations do not occur with a genuine delay; they occur when the delayed and undelayed situations are mixed. In the case of (1), with $n=1$, these phenomena, and several related phenomena, have been discussed at length by S. B. Norkin [14, Chapter II, §5]. Theorem 3 says that, in our circumstances, the two traditional definitions of oscillation coalesce.

If $g$ is in $G$, it is clear that there are solutions of (2) with each of $u, u^{\prime}, \ldots, u^{(2 n+1)}$ eventually positive. We shall call such solutions strongly increasing. Strongly increasing solutions of (3) are defined analogously. Our next theorem ensures the existence of solutions $u$ such that neither $u$ nor $-u$ is strongly increasing.

THEOREM 4. Suppose $g$ is in $G, b>0$, and $x$ is a continuous real-valued function on $[0, b]$. Then there is a solution $u$ of (2) such that $u(t)=x(t)$ if $0 \leqslant t \leqslant b$ and such that neither $u$ nor $-u$ is strongly increasing. The same conclusions are true with respect to (3).

For (3), with $n=1$, Theorem 4 is a 1esult of G. A. Kamenskir [5]. Ladas, Lakshmikantham, and Papadakis $[8, \S 4]$ have claimed Theorem 4 without proof, referring to Kamenskirs methods. Since Kamenskii’s paper is not readily available, we shall include a proof of Theorem 4. (We do not know if our methods are those of Kamenskii's, but we expect they are, inasmuch as Theorem 4 admits a fairly "natural" proof.)

Proof of Theorem 1. Suppose $g(c)<c$. Now there is $\delta>0$ such that $g(c+\delta)<c$, and there is $\xi$ in $(g(c+\delta), c)$ such that $u(\xi) \neq 0$. Let $b \geqslant c$ be such that $g(b)=\xi$. Now some higher derivative of $u$ is nonzero at $b$, contradicting the fact that $u(t)=0$ if $t \geqslant c$. The proof is complete.

Corollary 1 is obvious.

Proof of THEOREM 2. Suppose $u$ is a solution of (1), and suppose $b_{u} \leqslant c<d$ and $u(t)=0$ whenever $c \leqslant t \leqslant d$ (the proofs for (2), (3) and (4) are similar, and we shall not include them). Let $\left\{\gamma_{m}\right\}_{m=0}^{\infty}$ and $\left\{\delta_{m}\right\}_{m=0}^{\infty}$ be sequences such that $\gamma_{0}=c, \delta_{0}=d, \gamma_{m+1}=g\left(\gamma_{m}\right)$ and $\delta_{m+1}=g\left(\delta_{m}\right)$ if $m$ $>0$. Now each of $\left\{\gamma_{m}\right\}_{m=0}^{\infty}$ and $\left\{\delta_{m}\right\}_{m=0}^{\infty}$ is decreasing and bounded below, so they have limits. Since these limits are fixed points of $g$, they are both zero. Suppose $b_{u}=0$. Now $u(t)=0$ if $m$ is a nonnegative integer and $\gamma_{m} \leqslant t$ $<\delta_{m}$, so $u\left(b_{u}\right)=0$ since $u$ is continuous. But this is a contradiction. Suppose $b_{u}>0$. Let $k$ be the largest integer with $\gamma_{k} \geqslant b_{u}$. Now $g\left(b_{u}\right) \leqslant \gamma_{k+1}<b_{u}$. But $u\left(\gamma_{k+1}\right)=-u^{(2 n)}\left(\gamma_{k}\right) / q\left(\gamma_{k+1}\right)=0$ since $u(t)=0$ whenever $\gamma_{k} \leqslant t \leqslant \delta_{k}$. This is a contradiction and the proof is complete. 
Proof of Theorem 3. We shall prove Theorem 3 for 11 , . id thi inl er cases will be clear. It suffices to assume that $u$ is oscillatory, evt ntually nonnegative, but not eventually trivial, and pre juce a contradiction. Find $c \geqslant b_{u}$ such that $u(t) \geqslant 0$ if $t \geqslant c$, and find $d \geqslant c$ such that $g(t) \geqslant c$ if $t \geqslant d$. Now $u^{(2 n)}<0$ on $[d, \infty)$, so $u^{(2 n-1)}$ is nonincreasing on $[d, \infty)$. If $u^{(2 n-1)}$ is eventually trivial, then there is some point past which $u$ is a polynomial. But no nontrivial polynomial is oscillatory, so $u^{(2 n-1)}$ is not eventually trivial. Thus $u^{(2 n-1)}$ is either eventually positive or eventually negative. Similar arguments now show that $u^{(2 n-2)}$ is either eventually positive or eventually negative. Continuing this we see that $u$ is either eventually positive or eventually negative. But since we know $u$ is eventually nonnegative, this says $u$ is eventually positive, and, in particular, nonoscillatory. This contradiction cumpletes the proof.

Before proving Theorem 4 we need a lemma. The lemma is obvious, and we shall not include a proof.

LEMMA 1. Suppose $g$ is in $G$ and $u$ is a solution of (2). Suppose also that there is $c>b_{u}$ such that $u(t)>0$ if $g(c)<t \leqslant c$ and such that $u^{(k)}(c)>0$ if $k=1, \ldots, 2 n$. Then $u$ is strongly increasing. The analogous conclusion holds with respect to (3).

Proof of Theorem 4. If $-1 \leqslant \alpha \leqslant 1$ and $-1 \leqslant \beta \leqslant 1$, let $V(\alpha, \beta)$ be that solution $v$ of (2) such that $v(t)=\alpha x(t)$ if $0 \leqslant t \leqslant b, v^{(k)}(b)=0$ if $k=1$, $\ldots, 2 n-1$, and $v^{(2 n)}(b)=\beta$. If neither of $V(1,1)$ and $-V(1,1)$ is strongly increasing, we are through. Suppose, without loss in generality, that $V(1,1)$ is strongly increasing. Now $V(-1,-1)=-V(1,1)$, by linearity, so $-V(-1,-1)$ is strongly increasing. If neither of $V(1,-1)$ and $-V(1,-1)$ is strongly increasing, we are through, so suppose $V(1,-1)$ is strongly increasing. Lemma 1 and continuous dependence now say there are $\gamma$ and $\delta,-1<\delta<\gamma<1$, such that if $\gamma<\alpha \leqslant 1$ then $V(\alpha,-1)$ is strongly increasing and if $-1<\alpha$ $<\delta$ then $-V(\alpha,-1)$ is strongly increasing, and such that $\gamma$ is the smallest such number and $\delta$ is the largest such number. In particular, if $\delta \leqslant \eta<\gamma$, neither $V(\eta,-1)$ or $-V(\eta,-1)$ is strongly increasing. Since $-V(0,-1)$ is strongly increasing, the interval $[\delta, \gamma]$ does not contain zero. Thus $V(1,-1 / \delta)$ $=(1 / \delta) V(\delta,-1)$ satisfies the conclusions of the theorem. If $-V(1,-1)$ is strongly increasing, we can find $\eta,-1<\eta<1$, such that neither of $V(1, \eta)$ and $-V(1, \eta)$ is strongly increasing. The proof for (2) is complete, and the proof for (3) is analogous.

III. Equation (1). Since all of our results for (1) are known if $n=1$ (see [2], [12] and [20]), and for notational convenience, we assume in this section that $n>1$. The following three theorems are our primary results regarding (1). 
THEOREM 5. Statements (i) and (ii) are equivalent.

(i) $\int_{0}^{\infty} q(t) d t=\infty$.

(ii) If $g$ is in $G$ then every solution of (i) is oscillatory. Furthermore, if (i) fails, if $0<\alpha<1 /(2 n-1)$, if $g$ is in $G$, and if $g(t) \leqslant t^{\alpha}$ and

$$
g(t) \leqslant\left(\int_{t}^{\infty} q(s) d s\right)^{-\alpha}
$$

whenever $t \geqslant 0$, then (1) has a nonoscillatory solution.

THEOREM 6. Suppose $h$ and $g$ are in $G, b \geqslant 0, h(t) \leqslant g(t)$ whenever $t>b$, and (1) has a nonoscillatory solution. Then

$$
v^{(2 n)}(t)+q(t) v(h(t))=0
$$

has a nonoscillatory solution.

THEOREM 7. Suppose $g$ is a continuously differentiable member of $G$ and $\boldsymbol{g}^{\prime}(t) \leqslant 1$ whenever $t \geqslant 0$. If

$$
\int_{0}^{\infty}[g(s)]^{2 n-2} q(s) d s=\infty,
$$

or if (10) fails but every solution of

$$
w^{\prime \prime}(t)+\left(\frac{g^{\prime}(t)}{(2 n-3) !} \int_{t}^{\infty}[g(s)-g(t)]^{2 n-3} q(s) d s\right) w(g(t))=0
$$

is oscillatory, then every solution of (1) is oscillatory.

COROLLARY 2. If (5) has a nonoscillatory solution and $g$ is in $G$, then (1) has a nonoscillatory solution.

COROLlaRY 3. Suppose $g$ is a continuously differentiable member of $G$ and $0<g^{\prime}(t) \leqslant 1$ if $t \geqslant 0$. If (10) fails, and

$$
\left(\frac{z^{\prime}(t)}{g^{\prime}(t)}\right)^{\prime}+\left(\frac{g^{\prime}(t)}{(2 n-3) !} \int_{t}^{\infty}[g(s)-g(t)]^{2 n-3} q(s) d s\right) z(t)=0
$$

is oscillatory, then every solution of (1) is oscillatory.

Since it is known (see G. V. Anan'eva and V. I. Balaganskii [1], H. C. Howard [4], I. T. Kiguradze [6], V. A. Kondrat'ev [7], and C. A. Swanson [19, p. 175]) that if

$$
\int_{0}^{\infty} t^{2 n-2} q(t) d t=\infty
$$

then every solution of (5) is oscillatory, Theorem 5 makes it clear that oscillation of every solution of (5) is a much weaker condition on $q$ than is (ii). 
Theorem 6 and Corollary 2 (note that Corollary 2 is an immediate consequence of Theorem 6) illustrate our point that, in the relationship between (5) and (1), delays can destroy, but not induce, oscillation. Theorem 7 is an extension of [9, Theorem 1], and is known if $g$ is given by $g(t)=t$.

Proof of Theorem 5. Suppose (i) is true, $g$ is in $G$, and $u$ is a nonoscillatory solution of (1). If $u$ is eventually negative we may replace $u$ by $-u$, so we assume $u$ is eventually positive. Find $a \geqslant 0$ such that $u(t)>0$ if $t>a$, and find $b \geqslant a$ such that $g(t) \geqslant a$ if $t \geqslant b$. Now $u^{(2 n)}<0$ on $[b, \infty)$, so $u^{(2 n-1)}$ is eventually one-signed. Since $u^{(2 n-1)}$ is eventually one-signed, $u^{(2 n-2)}$ is eventually one-signed. Continuing this, we see that there is $c>b$ such that none of $u, u^{\prime}, \ldots, u^{(2 n-1)}$ has a zero in $[c, \infty)$. Let $j$ be the largest integer such that $u^{(i)}>0$ on $[c, \infty)$ if $i \leqslant j$. Since $u^{(2 n)}<0, j \neq 2 n$. Since $u^{(j)}>0$ and $u^{(j+1)}<0, u^{(j)}$ is bounded. Thus, if $j \leqslant k \leqslant 2 n-1, u^{(k)} u^{(k+1)}<0$ on $[c, \infty)$. Since $u^{(2 n)}<0$, this says that if $j \leqslant k \leqslant 2 n-1$ then $u^{(k)}>0$ on $[c, \infty)$ if $k$ is odd and $u^{(k)}<0$ on $[c, \infty)$ if $k$ is even. Thus $j$ is odd. Find $d>c$ such that $g(t)>c$ if $t>d$. Now, if $t>d, u(g(d))<u(g(t))$, since $u^{\prime}>0$ on $[c, \infty)$. Thus, if $t \geqslant d$,

$$
\begin{aligned}
u^{(2 n-1)}(d) & =u^{(2 n-1)}(t)+\int_{d}^{t} q(s) u(g(s)) d s \\
& \geqslant \int_{d}^{t} q(s) u(g(s)) d s \geqslant u(g(d)) \int_{d}^{t} q(s) d s,
\end{aligned}
$$

so

$$
u^{(2 n-1)}(d) \geqslant u(g(d)) \int_{d}^{\infty} q(s) d s .
$$

But this contradicts (i), so the proof of (i) $\rightarrow$ (ii) is complete.

Now suppose (i) fails. We shall show that (ii) fails. Let $\alpha$ be a positive number with $\alpha(2 n-1)<1$, and let $h$ in $G$ be given by

$$
h(t)=\min \left\{t,\left(\int_{t}^{\infty} q(s) d s\right)^{-1}\right\}
$$

Now

$$
\int_{0}^{\infty}[h(t)]^{\alpha(2 n-1)} q(t) d t<\infty .
$$

Let $a$ be a positive number, $a<1$, and find a positive number $b$ such that

$$
a+\left((1-\alpha) b+a^{1-\alpha}\right)^{\alpha /(1-\alpha)} b<1
$$

and 


$$
a-\left((1-\alpha) b+a^{1-\alpha}\right)^{\alpha /(1-\alpha)} b>0 .
$$

Let $k=\left((1-\alpha) b+a^{1-\alpha}\right)^{1 /(1-\alpha)}$. From (13) there is $c \geqslant 0$ such that

$$
\int_{c}^{\infty}[h(t)]^{\alpha(2 n-1)} q(t) d t \leqslant b .
$$

Let $x$ be a solution of

$$
x^{(2 n)}(t)+q(t)|x(h(t))|^{\alpha} \operatorname{sgn}(x(g(t)))=0
$$

for $t>c$ such that $x(t)=0$ if $0 \leqslant t \leqslant c, x^{(k)}(c)=0$, if $1 \leqslant k \leqslant 2 n-2$, and $x^{(2 n-1)}(c)=a$. Now, if $t \geqslant c,(16)$ says

$$
x(t)=a \frac{(t-c)^{2 n-1}}{(2 n-1) !}-\frac{1}{(2 n-1) !} \int_{c}^{t}(t-s)^{2 n-1} q(s)|x(h(s))|^{\alpha} \operatorname{sgn}(x(h(s))) d s,
$$

so

$$
|x(t)| \leqslant a t^{2 n-1}+t^{2 n-1} \int_{c}^{t} q(s)|x(h(s))|^{\alpha} d s .
$$

Let $y$ be given on $[c, \infty)$ by

$$
y(t)=a+\int_{c}^{t} q(s)|x(h(s))|^{\alpha} d s,
$$

and note that $y$ is nondecreasing. Also, (17) gives $|x(t)| \leqslant t^{2 n-1} y(t)$ if $t \geqslant c$. If $d \geqslant c$ and $h(d)=c$, then

$$
|x(h(t))| \leqslant[h(t)]^{2 n-1} y(h(t)) \leqslant[h(t)]^{2 n-1} y(t)
$$

whenever $t \geqslant d$. But clearly

$$
|x(h(t))| \leqslant[h(t)]^{2 n-1} y(t)
$$

if $c \leqslant t \leqslant d$, so (18) holds whenever $t \geqslant c$. If $t>c$ then

$$
y^{\prime}(t)=q(t)|x(h(t))|^{\alpha} \leqslant q(t)[h(t)]^{\alpha(2 n-1)} y(t)^{\alpha},
$$

so

$$
y(t) \leqslant\left(a^{1-\alpha}+(1-\alpha) \int_{c}^{t}[h(s)]^{\alpha(2 n-1)} q(s) d s\right)^{1 /(1-\alpha)} \leqslant k .
$$

Now (18) and (19) say

$$
|x(h(t))| \leqslant k[h(t)]^{2 n-1}
$$

if $t \geqslant c$. Now, if $t \geqslant c$, 


$$
x^{(2 n-1)}(t)=a-\int_{c}^{t} q(s)|x(h(s))|^{\alpha} \operatorname{sgn}(x(h(s))) d s,
$$

so (20) and (13) say that $\lambda=\lim _{t \rightarrow \infty} x^{(2 n-1)}(t)$ exists. Also, $a-k^{\alpha} b<\lambda$ $<a+k^{\alpha} b$, so $0<\lambda<1$. Since $\lim _{t \rightarrow \infty} x(t) / t^{2 n-1}=\lambda /(2 n-1) !=\mu>0$, we see $x(t) \rightarrow \infty$ as $t \rightarrow \infty$. Also, since $x(c)=0$ and $x(t) \rightarrow \infty$ as $t \rightarrow \infty$, there is a largest number $m$ in $[c, \infty)$ such that $x(h(m))=1$. Note that the fact that (13) implies (16) has a solution $x$ for which $\lim _{t \rightarrow \infty} x^{(2 n-1)}(t)$ exists and is positive follows from the work of T. Kusano and H. Onose [21, Theorem 1]. Furthermore, our proof is virtually the same as that in [21]. We have reproduced it here to point out that $\mu$ can be chosen with $\mu<1$, and $x$ can be chosen with the equation $x(h(t))=1$ having a solution. If $t>c$ then

$$
\begin{aligned}
x^{\prime}(t) & =a \frac{(t-c)^{2 n-2}}{(2 n-2) !}-\frac{1}{(2 n-2) !} \int_{c}^{t}(t-s)^{2 n-2} q(s)|x(h(s))|^{\alpha} \operatorname{sgn}(x(h(s))) d s \\
& >a \frac{(t-c)^{2 n-2}}{(2 n-2) !}-\frac{(t-c)^{2 n-2}}{(2 n-2) !} \int_{c}^{t} q(s)|x(h(s))|^{\alpha} d s \\
& >\frac{(t-c)^{2 n-2}}{(2 n-2) !}\left(a-k^{\alpha} \int_{c}^{t} q(s)[h(s)]^{\alpha(2 n-1)} d s\right) \\
& >\frac{(t-c)^{2 n-2}}{(2 n-2) !}\left(a-k^{\alpha} b\right)>0 .
\end{aligned}
$$

Thus $x^{\prime}>0$ on $(c, \infty)$, so $x$ is increasing on $[c, \infty)$. Clearly now there is a member $f$ of $G$ such that $f(t)=h(t)$ if $t<m$ and $x(f(t))=x(h(t))^{\alpha}$ if $t>m$. Now $x$ is a solution of

$$
x^{(2 n)}(t)+q(t) x(f(t))=0,
$$

and it is clear that (ii) fails. Also, $x(f(t)) / f(t)^{2 n-1} \rightarrow \mu$ and $x(h(t)) / h(t)^{2 n-1}$ $\rightarrow \mu$ as $t \rightarrow \infty$, so

$$
\lim _{t \rightarrow \infty} \frac{f(t)^{2 n-1}}{h(t)^{\alpha(2 n-1)}}=\lim _{t \rightarrow \infty} \frac{f(t)^{2 n-1}}{x(f(t))} \frac{x(h(t))^{\alpha}}{h(t)^{\alpha(2 n-1)}}=\frac{\mu^{\alpha}}{\mu}>1,
$$

since $\mu<1$, so $\lim _{t \rightarrow \infty} f(t) / h(t)^{\alpha}>1$. Find a number $\beta>m$ such that $f(t) / h(t)^{\alpha}>1$ whenever $t>\beta$. Now $h(t)^{\alpha}<f(t)$ if $t>\beta$. It is now clear that if the proof of Theorem 6 is independent of Theorem 5 then the last conclusion of Theorem 5 follows from Theorem 6. The proof of Theorem 6 is independent of Theorem 5.

ProOF OF THEOREM 6. Let $u$ be an eventually positive solution of (1). Let $c \geqslant b$ be such that none of $u, u^{\prime}, \ldots, u^{(2 n-1)}$ has a zero on $[c, \infty)$, and let $j$ be the largest integer such that $u^{(i)}>0$ on $[c, \infty)$ if $i<j$. Find $d>c$ such that 
if $t>d$ then $h(t) \geqslant c$. If $t \geqslant d$ then

and

$$
u(t)>u(d)+\frac{1}{(j-1) !} \int_{d}^{t}(t-s)^{j-1} u^{(j)}(s) d s
$$

$$
u^{(j)}(t) \geqslant \frac{1}{(2 n-j-1) !} \int_{t}^{\infty}(s-t)^{2 n-j-1} q(s) u(g(s)) d s
$$

so

$$
\begin{aligned}
u(t) \geqslant & u(d)+\frac{1}{(j-1) !(2 n-j-1) !} \\
& \cdot \int_{d}^{t}(t-s)^{j-1}\left(\int_{s}^{\infty}(\xi-s)^{2 n-j-1} q(\xi) u(g(\xi)) d \xi\right) d s \\
\geqslant & u(d)+\frac{1}{(j-1) !(2 n-j-1) !} \\
& \cdot \int_{d}^{t}(t-s)^{j-1}\left(\int_{s}^{\infty}(\xi-s)^{2 n-j-1} q(\xi) u(h(\xi)) d \xi\right) d s
\end{aligned}
$$

since $u^{\prime}>0$ and $u$ is increasing on $[c, \infty)$. Now (21) and standard iteration methods say that there is a continuous real-valued function $v$ on $[0, \infty)$ such that $v(t)=u(t)$ if $t \geqslant d$, such that $u(d)<v(t)<u(t)$ if $t \geqslant d$, and such that

$$
\begin{aligned}
v(t)= & u(d)+\frac{1}{(j-1) !(2 n-j-1) !} \\
& \cdot \int_{d}^{t}(t-s)^{j-1}\left(\int_{s}^{\infty}(\xi-s)^{2 n-j-1} q(\xi) v(h(\xi)) d \xi\right) d s
\end{aligned}
$$

if $t>d$. Differentiation of (22) says $v$ is a solution of (9), and clearly $v$ is nonoscillatory, so the proof is complete.

ProOf OF THEOREM 7. Let $u$ be an eventually positive solution of (1), find $c>b_{u}$ such that none of $u, u^{\prime}, \ldots, u^{(2 n-1)}$ has a zero in $[c, \infty)$, and let $j$ be the largest integer such that $u^{(i)}>0$ on $[c, \infty)$ if $i<j$. Find $d>c$ such that $g(t) \geqslant c$ if $t>d$. Suppose $j>1$. If $t>d$ then

$$
u^{(j)}(t) \geqslant \frac{1}{(2 n-j-1) !} \int_{t}^{\infty}(s-t)^{2 n-j-1} q(s) u(g(s)) d s .
$$

Also, if $\sigma \geqslant g(d)$,

$$
u(\sigma) \geqslant \frac{1}{(j-2) !} \int_{g(d)}^{\sigma}(\sigma-\xi)^{j-2} u^{(j-1)}(\xi) d \xi,
$$

so if $s>d$, 
(23)

$$
u(g(s))>\frac{1}{(j-2) !} \int_{g(d)}^{g(s)}[g(s)-\xi] \cdots{ }^{(j-1)}(\xi) d \xi .
$$

Since $g^{\prime}(t)<1$ whenever $t \geqslant 0, g(s)-g(t)<s-t$ whenever $0<t<s$, and ince $u^{(j)}>0$ on $[c, \infty), u^{(j-1)}$ is increasing on $[c, \infty)$. Thus, if $t>d$,

$$
\begin{aligned}
u^{(j)}(t) & \geqslant \frac{1}{(2 n-3) !} \int_{t}^{\infty}(s-t)^{2 n-j-1} q(s)\left(\int_{g(d)}^{g(s)}[g(s)-\xi]^{j-2} u^{(j-1)}(\xi) d \xi\right) d s \\
& >\frac{1}{(2 n-3) !} \int_{t}^{\infty}(s-t)^{2 n-j-1} q(s)\left(\int_{g(t)}^{g(s)}[g(s)-\xi]^{j-2} u^{(j-1)}(\xi) d \xi\right) d s \\
& >\frac{u^{(j-1)}(g(t))}{(2 n-3) !} \int_{t}^{\infty}(s-t)^{2 n-j-1} q(s)\left(\int_{\xi(t)}^{g(s)}[g(s)-\xi]^{j-2} d \xi\right) d s \\
& =\frac{u^{(j-1)}(g(t))}{(2 n-3) !(j-1)} \int_{t}^{\infty}(s-t)^{2 n-j-1} q(s)[g(s)-g(t)]^{j-1} d s \\
& >\frac{u^{(j-1)}(g(t))}{(2 n-3) !(j-1)} \int_{t}^{\infty}[g(s)-g(t)]^{2 n-2} q(s) d s .
\end{aligned}
$$

In particular,

$$
u^{(j)}(d)>\frac{u^{(j-1)}(g(d))}{(2 n-3) !(j-1)} \int_{d}^{\infty}[g(s)-g(d)]^{2 n-2} q(s) d s,
$$

so

$$
\int_{d}^{\infty}[g(s)-g(d)]^{2 n-2} q(s) d s<\infty
$$

If $j=1$,

$$
\begin{aligned}
u^{\prime}(t) & >\frac{1}{(2 n-2) !} \int_{t}^{\infty}(s-t)^{2 n-2} q(s) u(g(s)) d s \\
& >\frac{u(g(t))}{(2 n-2) !} \int_{t}^{\infty}[g(s)-g(t)]^{2 n-2} q(s) d s,
\end{aligned}
$$

so (24) holds in either case. But (24) implies the failure of (10), so half the proof is complete.

Suppose $j=2 n-1$. Now

$$
\begin{aligned}
u^{(2 n-1)}(t) & \geqslant \int_{t}^{\infty} q(s) u(g(s)) d s \\
& \geqslant \frac{1}{(2 n-3) !} \int_{t}^{\infty} q(s)\left(\int_{g(d)}^{g(s)}[g(s)-\xi]^{2 n-3} u^{(2 n-2)}(\xi) d \xi\right) d s \\
& \geqslant \frac{1}{(2 n-3) !} \int_{t}^{\infty} q(s)\left(\int_{g(t)}^{g(s)}[g(s)-\xi]^{2 n-3} u^{(2 n-2)}(\xi) d \xi\right) d s
\end{aligned}
$$


if $t \geqslant d$. But this and standard iteration methods say there is a continuous real-valued function $w$ on $[0, \infty)$ such that $w(t)=u^{(2 n-2)}(t)$ if $c \leqslant t \leqslant d$, $u^{(2 n-2)}(d) \leqslant w(t) \leqslant u^{(2 n-2)}(t)$ if $t \geqslant d$ and

$$
w^{\prime}(t)=\frac{1}{(2 n-3) !} \int_{t}^{\infty} q(s)\left(\int_{g(t)}^{g(s)}[g(s)-\xi]^{2 n-3} w(\xi) d \xi\right) d s
$$

if $t>d$. Now differentiation of (25) yields (11), so $w$ is a nonoscillatory solution of (11). This completes the proof if $j=2 n-1$. It should be noted that the case $j=2 n-1$ is the only place we actually need that $g$ be differentiable with $g^{\prime} \leqslant 1$. In all other places it suffices to require that $g(s)-g(t)<s-t$ if $0 \leqslant t<s$. To complete our proof we shall actually prove the stronger conclusion that if $j<2 n-1$ then there is a nonoscillatory solution of

$$
w^{\prime \prime}(t)+\left(\frac{1}{(2 n-3) !} \int_{t}^{\infty}[g(s)-g(t)]^{2 n-3} q(s) d s\right) w(g(t))=0 .
$$

Suppose $j<2 n-1$. If $j>1$ and $t \geqslant d$,

$$
\begin{aligned}
-u^{(j+1)}(t)=\frac{1}{(2 n-j-2) !} \int_{t}^{\infty}(s-t)^{2 n-j-2} q(s) u(g(s)) d s \\
\quad>\frac{1}{(2 n-j-2) !(j-2) !} \\
\quad \cdot \int_{t}^{\infty}(s-t)^{2 n-j-2} q(s)\left(\int_{g(d)}^{g(s)}[g(s)-\xi]^{j-2} u^{(j-1)}(\xi) d \xi\right) d s \\
>\frac{1}{(2 n-j-2) !(j-2) !} \\
\quad \cdot \int_{t}^{\infty}(s-t)^{2 n-j-2} q(s)\left(\int_{g(t)}^{g(s)}[g(s)-\xi]^{j-2} u^{(j-1)}(\xi) d \xi\right) d s \\
>\frac{u^{(j-1)}(g(t))}{(2 n-j-2) !(j-2) !} \int_{t}^{\infty}(s-t)^{2 n-j-2} q(s)\left(\int_{g(t)}^{g(s)}[g(s)-\xi]^{j-2} d \xi\right) d s \\
\quad=\frac{u^{(j-1)}(g(t))}{(2 n-j-2) !(j-1) !} \int_{t}^{\infty}(s-t)^{2 n-j-2} q(s)[g(s)-g(t)]^{j-1} d s \\
>\frac{u^{(j-1)}(g(t))}{(2 n-3) !} \int_{t}^{\infty}[g(s)-g(t)]^{2 n-3} q(s) d s .
\end{aligned}
$$

If $\phi$ is given by 


$$
\phi(t)=\frac{1}{(2 n-3) !} \int_{t}^{\infty}[g(s)-g(t)] " ' q(s) d s,
$$

this says $u^{(j+1)}(t)+\phi(t) u^{(j-1)}(g(t)) \leqslant 0$. If $j=1$ and $t \geqslant d$,

$$
-u^{\prime \prime}(t)=\frac{1}{(2 n-3) !} \int_{t}^{\infty}(s-t)^{2 n-3} q(s) u(g(s)) d s>u(g(t)) \phi(t),
$$

so in either case we have a positive solution of

$$
v^{\prime \prime}(t)+\phi(t) v(g(t))<0
$$

if $t>d$. Since $v>0$ and $v^{\prime \prime}<0$ on $[d, \infty), v^{\prime}>0$ on $[d, \infty)$. If $\tau>t>d$,

$$
\begin{gathered}
v^{\prime}(\tau)-v^{\prime}(t)<-\int_{t}^{\tau} \phi(s) v(g(s)) d s, \\
v^{\prime}(t)>v^{\prime}(\tau)+\int_{t}^{\tau} \phi(s) v(g(s)) d s>\int_{t}^{\tau} \phi(s) v(g(s)) d s,
\end{gathered}
$$

so $v^{\prime}(t)>\int_{t}^{\infty} \phi(s) v(g(s)) d s$. Now there is $w$ such that $w(t)=v(t)$ if $c<t$ $\langle d, v(d)<w(t)<v(t)$ if $t>d$, and

$$
w^{\prime}(t)=\int_{t}^{\infty} \phi(s) w(g(s)) d s .
$$

Differentiation of (28) yields (26), and the proof is complete.

Proof of Corollary 3. Suppose (10) fails, and let $\phi$ be given on $[0, \infty)$ by

$$
\phi(t)=\frac{g^{\prime}(t)}{(2 n-3) !} \int_{t}^{\infty}[g(s)-g(t)]^{2 n-3} q(s) d s .
$$

It suffices to show that if (11) has an eventually positive solution then (12) has an eventually positive solution. Suppose $w$ is an eventually positive solution of (11). Find $c$ such that if $t>c$ then (11) holds and $w(t)>0$, and find $d>c$ such that if $t>d$ then $g(t)>c$. Now

$$
w^{\prime}(t) \geqslant \int_{t}^{\infty} \phi(s) w(g(s)) d s
$$

if $t>d$. Find $\alpha>d$ such that $g(t)>d$ if $t>\alpha$. Now

$$
w^{\prime}(g(t)) \geqslant \int_{g(t)}^{\infty} \phi(s) w(g(s)) d s>\int_{t}^{\infty} \phi(s) w(g(s)) d s
$$

if $t>\alpha$, so

$$
g^{\prime}(t) w^{\prime}(g(t)) \geqslant g^{\prime}(t) \int_{t}^{\infty} \phi(s) w(g(s)) d s
$$

if $t>\alpha$. Let $y$ be given on $[\alpha, \infty)$ by $y(t)=w(g(t))$. Now 


$$
y^{\prime}(t) \geqslant g^{\prime}(t) \int_{t}^{\infty} \phi(s) y(s) d s .
$$

Standard iteration methods now say that there is a continuously differentiable function $z$ from $[\alpha, \infty)$ to $[y(\alpha), \infty)$ such that $z(\alpha)=y(\alpha), z(t) \leqslant y(t)$ if $t \geqslant \alpha$, and

$$
z^{\prime}(t)=g^{\prime}(t) \int_{t}^{\infty} \phi(s) z(s) d s
$$

if $t>\alpha$. Clearly $z$ is a solution of $(12)$ on $[\alpha, \infty)$, and $z$ has no zeros on $[\alpha, \infty)$, so the proof is complete.

IV. Equation (2). Since we know that if $g$ is in $G$ then (2) has stron increasing solutions, the best one can hope for in ensuring oscillation in (2) is to ensure that every eventually positive suiution is strongly increasing.

THEOREM 8. Statements (iii) and (iv) are equivalent.

(iii) $\int_{0}^{\infty} t q(t) d t=\infty$.

(iv) If $g$ is in $G$ every eventually positive solution of (2) is strongly increasing.

THEOREM 9. Suppose $h$ and $g$ are in $G, b \geqslant 0, h(t) \leqslant g(t)$ whenever $t \geqslant b$, and (2) has an eventually positive solution which is not strongly increasing. Then

$$
v^{(2 n+1)}(t)-q(t) v(h(t))=0
$$

has an eventually positive solution which is not strongly increasing.

THEOREM 10. Suppose $g$ is in $G$ and $g(s)-g(t) \leqslant s-t$ whenever $0 \leqslant t \leqslant s$. If

$$
\int_{0}^{\infty}[g(s)]^{2 n-1} q(s) d s=\infty,
$$

or if (30) fails but every solution of

$$
w^{\prime \prime}(t)+\left(\frac{1}{(2 n-2) !} \int_{t}^{\infty}[g(s)-g(t)]^{2 n-2} q(s) d s\right) w(g(t))=0
$$

is oscillatory, then every eventually positive solution of (2) is strongly increasing.

One can draw corollaries from Theorems 8, 9 and 10 in much the same way that corollaries were drawn from Theorems 5, 6 and 7, and we leave this to the reader. The following corollary is an immediate consequence of Theorems 4 and 10.

COROLlary 4. Let $g$ be as in Theorem 10, and suppose that either (30) is true, or (30) fails and every solution of (31) is oscillatory. If $b>0$ and $x$ is a continuous real-valued function on $[0, b]$, then there is an oscillatory solution $u$ of (2) with $u(t)=x(t)$ whenever $0 \leqslant t \leqslant b$. 
Since it is known [11] that $\int_{0}^{\infty} t^{2 n-1} q(t) d t=\infty$ implies thal every eventually positive solution of (6) is strongly increasing, Theorem 8 makes it clear that to require that every eventually positive solution of (6) be strongly increasing is a much weaker condition on $q$ than is (iv). The proof that (iii) $\rightarrow$ (iv) is similar to the proof of (i) $\rightarrow$ (ii), so we shall only indicate the high points. The proof that (iv) $\rightarrow$ (iii), on the other hand, requires entirely new techniques, since the results of Kusano and Onose [21] cannot be used here.

Proof of Theorem 8. Suppose (iii) is true, $g$ is in $G$, and $u$ is an eventually positive solution of (2) which is not strongly increasing. Find $c>0$ such that (2) is true whenever $t \geqslant c$, and such that none of $u, u^{\prime}, \ldots, u^{(2 n)}$ has a zero in $[c, \infty)$. Let $j$ be the largest inte, er such that $u^{(i)}>0$ on $[c, \infty)$ if $i<j$, and note that $j$ is odd. Find $d \geqslant c$ such that $g(t) \geqslant c$ if $t>d$. By hypothesis, $j \neq 2 n+1$, so

$$
\begin{aligned}
u^{(j)}(d) & \geqslant \frac{1}{(2 n-j) !} \int_{d}^{\infty}(s-d)^{2 n-j} q(s) u(g(s)) d s \\
& \geqslant \frac{u(g(d))}{(2 n-j) !} \int_{d}^{\infty}(s-d)^{2 n-j} q(s) d s .
\end{aligned}
$$

Thus

$$
\int_{d}^{\infty}(s-d)^{2 n-j} q(s) d s<\infty,
$$

so $\int_{0}^{\infty} s^{2 n-j} q(s) d s<\infty$, and $\int_{0}^{\infty} t q(t) d t<\infty$ since $2 n-j>1$. This contradiction completes the proof of (iii) $\rightarrow$ (iv).

To complete the proof we shall show that if (iii) is false then (iv) is false. Suppose (iii) is false. Let $h$ be a member of $G$ such that

$$
\int_{0}^{\infty} \operatorname{th}(t) q(t) d t<\infty
$$

Let $v$ be given on $[0, \infty)$ by

$$
v(t)=1+\sum_{k=1}^{2 n-1} \frac{t^{k}}{k !}+\frac{1}{(2 n-2) !} \int_{0}^{t}(t-s)^{2 n-2}\left(\int_{s}^{\infty}(\xi-s) h(\xi) q(\xi) d \xi\right) d s .
$$

Now $v(t)>0$ and $h(t) \leqslant t<v(t)$ if $t \geqslant 0$. Also,

$$
\begin{aligned}
v^{(2 n-1)}(t) & =1+\int_{t}^{\infty}(s-t) h(s) q(s) d s, \\
v^{(2 n)}(t) & =-\int_{t}^{\infty} h(s) q(s) d s,
\end{aligned}
$$

and $v^{(2 n+1)}(t)=h(t) q(t)$ if $t \geqslant 0$. Let $p=v^{(2 n+1)} / v$. Now $v$ is a positive solution of 


$$
v^{(2 n+1)}(t)-p(t) v(t)=0
$$

which is not strongly increasing. Since $h$ is in $G$ there is at least number $c$ such that $h(c)=1$. Since $v$ is increasing and $h(t)<v(t)$ whenever $t \geqslant 0$, there is a member $g$ of $G$ such that $g(t)=0$ if $0 \leqslant t \leqslant c$ and $h(t)=v(g(t))$ if $t \geqslant c$. Now, if $t \geqslant c, p(t) v(t)=h(t) q(t)=q(t) v(g(t))$, so (32) says $v$ satisfies (2) on $(c, \infty)$. This completes the proof of Theorem 8 .

PrOOF OF THEOREM 9. Let $u$ be an eventually positive solution of (2) which is not strongly increasing. Find $c \geqslant 0$ such that (2) is true if $t \geqslant c$ and such that none of $u, u^{\prime}, \ldots, u^{(2 n)}$ has a zero in $[c, \infty)$, and find $d \geqslant c$ such that $h(t) \geqslant c$ if $t \geqslant d$. Now $j \neq 2 n+1$, since $u$ is not strongly increasing. Thus, if $t \geqslant d$,

and

$$
u(t) \geqslant u(d)+\frac{1}{(j-1) !} \int_{\alpha}^{t}(t-s)^{j-1} u^{(j)}(s) d s
$$

$$
u^{(j)}(t) \geqslant \frac{1}{(2 n-j) !} \int_{t}^{\infty}(s-t)^{2 n-j} q(s) u(g(s)) d s,
$$

so

$$
\begin{aligned}
u(t) \geqslant & u(d)+\frac{1}{(j-1) !(2 n-j) !} \\
& \quad \int_{d}^{t}(t-s)^{j-1}\left(\int_{s}^{\infty}(\xi-s)^{2 n-j} q(\xi) u(g(\xi)) d \xi\right) d s \\
\geqslant & u(d)+\frac{1}{(j-1) !(2 n-j) !} \int_{d}^{t}(t-s)^{j-1}\left(\int_{s}^{\infty}(\xi-s)^{2 n-j} q(\xi) u(h(\xi)) d \xi\right) d s .
\end{aligned}
$$

The remainder of the proof is so similar to the proof of Theorem 6 that we shall not include it.

It should be noted that (31) is more analogous to (26) then to (11), and that (11) is used in Theorem 3 instead of (26) only because of one case. The nature of (2) is such that the analogous case does not occur in Theorem 10.

Proof OF THEOREM 10. Let $u$ be an eventually positive solution of (2) which is not strongly increasing. Find $c \geqslant 0$ such that (2) is true if $t \geqslant c$ and such that none of $u, u^{\prime}, \ldots, u^{(2 n)}$ has a zero in $[c, \infty)$. Find $d \geqslant c$ such that $g(t) \geqslant c$ if $t \geqslant d$. Let $j$ be the largest integer such that $u^{(i)}>0$ on $[c, \infty)$ if $i \leqslant j$. Now $j$ is odd and $j \neq 2 n+1$. Suppose $j>1$. Now, if $t \geqslant d$,

$$
u^{(j)}(t) \geqslant \frac{1}{(2 n-j) !} \int_{t}^{\infty}(s-t)^{2 n-j} q(s) u(g(s)) d s .
$$

Also, if $s \geqslant d$, 


$$
u(g(s)) \geqslant \frac{1}{(j-2) !} \int_{g(d)}^{g(s)}[g(s)-\xi]^{j-2} u^{(j-1)}(\xi) d \xi,
$$

so

$$
\begin{aligned}
u^{(j)}(d) & >\frac{1}{(2 n-2) !} \int_{d}^{\infty}(s-d)^{2 n-j} q(s)\left(\int_{g(d)}^{g(s)}[g(s)-\xi]^{j-2} u^{(j-1)}(\xi) d \xi\right) d s \\
& >\frac{u^{(j-1)}(g(d))}{(2 n-2) !} \int_{d}^{\infty}(s-d)^{2 n-j} q(s)\left(\int_{g(d)}^{g(s)}[g(s)-\xi]^{j-2} d \xi\right) d s \\
& =\frac{u^{(j-1)}(g(d))}{(2 n-2) !(j-1)} \int_{d}^{\infty}(s-d)^{2 n-j}[g(s)-g(d)]^{j-1} q(s) d s \\
& >\frac{u^{(j-1)}(g(d))}{(2 n-2) !(j-1)} \int_{d}^{\infty}[g(s)-g(d)]^{2 n-1} q(s) d s,
\end{aligned}
$$

and

$$
\int_{d}^{\infty}[g(s)-g(d)]^{2 n-1} q(s) d s<\infty
$$

If $j=1$,

$$
\begin{aligned}
u^{\prime}(d) & \geqslant \frac{1}{(2 n-1) !} \int_{d}^{\infty}(s-d)^{2 n-1} q(s) u(g(s)) d s \\
& \geqslant \frac{u(g(d))}{(2 n-1) !} \int_{d}^{\infty}(s-d)^{2 n-1} q(s) d s \\
& \geqslant \frac{u(g(d))}{(2 n-1) !} \int_{d}^{\infty}[g(s)-g(d)]^{2 n-1} q(s) d s
\end{aligned}
$$

so (33) holds in either case. But (33) implies the failure of (30), so the first part of the proof is complete.

Again suppose $j>1$. Now, if $t>d$,

$$
\begin{aligned}
-u^{(j+1)}(t)= & \frac{1}{(2 n-j-1) !} \int_{t}^{\infty}(s-t)^{2 n-j-1} q(s) u(g(s)) d s \\
\geqslant & \frac{1}{(2 n-j-1) !(j-2) !} \int_{t}^{\infty}(s-t)^{2 n-j-1} q(s) \\
& \cdot\left(\int_{g(d)}^{g(s)}[g(s)-\xi]^{j-2} u^{(j-1)}(\xi) d \xi\right) d s \\
\geqslant & \frac{1}{(2 n-j-1) !(j-2) !} \int_{t}^{\infty}(s-t)^{2 n-j-1} q(s)
\end{aligned}
$$




$$
\begin{aligned}
& \cdot\left(\int_{g(t)}^{g(s)}[g(s)-\xi]^{j-2} u^{(j-1)}(\xi) d \xi\right) d s \\
\geqslant & \frac{u^{(j-1)}(g(t))}{(2 n-j-1) !(j-2) !} \int_{t}^{\infty}(s-t)^{2 n-j-1} q(s)\left(\int_{g(t)}^{g(s)}[g(s)-\xi]^{j-2} d \xi\right) d s \\
= & \frac{u^{(j-1)}(g(t))}{(2 n-j-1) !(j-1) !} \int_{t}^{\infty}(s-t)^{2 n-j-1} q(s)[g(s)-g(t)]^{j-1} d s \\
\geqslant & \frac{u^{(j-1)}(g(t))}{(2 n-2) !} \int_{t}^{\infty}[g(s)-g(t)]^{2 n-2} q(s) d s .
\end{aligned}
$$

If $\phi$ is given by

$$
\phi(t)=\frac{1}{(2 n-2) !} \int_{t}^{\infty}[g(s)-g(t)]^{2 n-2} q(s) d s,
$$

this says that $u^{(j+1)}(t)+\phi(t) u^{(j-1)}(g(t)) \leqslant 0$ if $t \geqslant d$. Suppose $j=1$. Now

$$
\begin{aligned}
-u^{\prime \prime}(t) & =\frac{1}{(2 n-2) !} \int_{t}^{\infty}(s-t)^{2 n-2} q(s) u(g(s)) d s \\
& \geqslant \frac{u(g(t))}{(2 n-2) !} \int_{t}^{\infty}[g(s)-g(t)]^{2 n-2} q(s) d s,
\end{aligned}
$$

so in either case we have a positive solution of $(27)$ on $[d, \infty)$. The remainder of the proof is similar to the proof of Theorem 7, and we desist.

V. Equation (3). As in §II, we assume throughout this section that $n>1$. It is known (see [11]) that (7) not only has solutions which are strongly increasing but also has solutions which are strongly decreasing, in the sense that $(-1)^{k} u^{(k)}(t)>0$ if $t \geqslant 0$ and $k=0,1, \ldots, 2 n-1$. The existence of strongly decreasing solutions is, at least with respect to our present concerns, the primary difference between (2) and (3).

Suppose $g$ is in $G$, and $u$ is an eventually positive solution of (3). Find $c \geqslant 0$ such that (3) is true if $t \geqslant c$ and such that none of $u, u^{\prime}, \ldots, u^{(2 n-1)}$ has a zero in $[c, \infty)$. Let $j$ be the largest integer such that $u^{(i)}>0$ on $[c, \infty)$ if $i \geqslant j$. Now $j$ is even. If $j=2 n$ then $u$ is strongly increasing, and if $j=0$ then $u$ is strongly decreasing. But if $j \neq 0$, then $j \geqslant 2$, so $u^{\prime}$ is positive and increasing, and $u$ is unbounded. Thus $u$ is bounded if and only if $j=0$. Ladas, Lakshmikantham, and Papadakis [8, Theorem 2.1] have shown that, if

$$
\limsup _{t \rightarrow \infty} \int_{g(t)}^{t}[g(t)-g(s)]^{2 n-1} q(s) d s>(2 n-1) !,
$$


then every bounded solution of (3) is oscillatory, i.e., " $j=0$ " is impossible in the above circumstance. (Actually, [8, Theorem 2.1] has $g$ continuously differentiable, but an examination of the proof shows that thi: is not necessary.) In \$III we singled out for special attention the eventually positive solutions of (2) which are strongly increasing. The above discussion makes it clear that the analogy here is the eventually positive unbounded solutions. which are strongly increasing. We offer tree theorems. The proofs are so similar to those of Theorems 8, 9 and 10 that we shall not include them.

THEOREM 11. Statements (iii) and (v) are equivalent.

(v) If $g$ is in $G$ every eventually positive unbounded solution of (3) is strongly increasing.

THEOREM 12. Suppose $h$ and $g$ are as in Theorem 9, and (3) has an eventually positive unbounded solution which is not strongly increasing. Then

$$
v^{(2 n)}(t)-q(t) v(h(t))=0
$$

has an eventually positive unbounded solution which is not strongly increasing.

Theorem 13. Suppose $g$ is in $G$ and $g(s)-g(t) \leqslant s-t$ whenever $0 \leqslant t \leqslant s$. If

$$
\int_{0}^{\infty}[g(s)]^{2 n-2} q(s) d s=\infty,
$$

or if (35) fails and every solution of

$$
w^{\prime \prime}(t)+\left(\frac{1}{(2 n-3) !} \int_{t}^{\infty}[g(s)-g(t)]^{2 n-3} q(s) d s\right) w(g(t))=0
$$

is oscillatory, then every eventually positive unbounded solution of (3) is strongly increasing.

The aforementioned result of Ladas, Lakshmikantham, and Papadakis [8] can be coupled with Theorem 13 to produce the following result.

COROLlARY 5. If (34) and (35) are true, or if (34) is true, (35) is false, and every solution of (36) is oscillatory, then every eventually positive solution of (3) is strongly increasing.

Theorem 4 and Corollary 5 can be combined to give a corollary similar to Corollary 4 , and we desist.

VI. Equation (4). It follows from a result of P. Hartman and A. Wintner [3] that there exists a strongly decreasing solution of (8). On the other hand, it is known [12] that if $q$ is "large" enough then strongly decreasing solutions of (8) 
are the only eventually positive solutions of (8), i.e., there are no unbounded nonoscillatory solutions.

Suppose $g$ is in $G$ and $u$ is an eventually positive solution of (4). Find $c \geqslant 0$ such that (4) is true if $t \geqslant c$ and such that none of $u, u^{\prime}, \ldots, u^{(2 n)}$ has a zero in $[c, \infty)$. Let $j$ be the largest integer such that $u^{(i)}>0$ on $[c, \infty)$ if $i \leqslant j$. Now $j$ is even. Thus $u$ is unbounded if and only if $j>0$. With the restriction " $j>0$ " in the proofs, virtually all of the analysis of $\$$ II can be performed on (4) instead of (1). The following theorems are so similar to Theorems 5, 6 and 7 that we do not include proofs.

THEOREM 14. Statements (i) and (vi) are equivalent.

(vi) If $\mathbf{g}$ is in $G$ every eventually positive solution of (4) is bounded.

THEOREM 15. Suppose $h$ and $g$ are as in Theorem 6, and (4) has no eventually positive unbounded solution. Then

$$
v^{(2 n+1)}(t)+q(t) v(h(t))=0
$$

has an eventually positive unbounded solution.

THEOREM 16. Let $g$ be as in Theorem 3. If

$$
\int_{0}^{\infty}[g(s)]^{2 n-1} q(s) d s=\infty,
$$

or if (37) fails but every solution of

$$
w^{\prime \prime}(t)+\left(\frac{g^{\prime}(t)}{(2 n-2) !} \int_{t}^{\infty}[g(s)-g(t)]^{2 n-2} q(s) d s\right) w(g(t))=0
$$

is oscillatory, then (4) has no eventually positive unbounded solutions.

It follows from [8, Theorem 2.1] that if

$$
\limsup _{t \rightarrow \infty} \int_{g(t)}^{t}[g(t)-g(s)]^{2 n} q(s) d s>(2 n) !
$$

then (4) has no bounded nonoscillatory solutions. Thus we have the following result.

COROLlaRy 6. If (37) and (39) are true, or if (37) is false, every solution of (38) is oscillatory, and (39) is true, then every solution of (4) is oscillatory.

\section{REFERENCES}

1. G. V. Anan'eva and V. I. Balaganskĩ, Oscillation of the solutions of certain differential equations of high order, Uspehi Mat. Nauk 14 (85) (1959), 135-140. MR 21 \# 1428. (Russian)

2. T. Burton and $R$. Grimmer, Oscillation, continuation, and uniqueness of solutions of retarded differential equations, Trans. Amer. Math. Soc. 179 (1973), 193-209. MR 48 \#2523. 
3. P. Hartman and A. Wintner, Linear differential and differenc. ¿cations with monotone solütions, Amer. J. Math. 75 (1953), 731-743. MR 15, 221.

4. H. C. Howaid, Oscillation criteria for even order differential equations, Ann. Mat. Pura Appl. (4) 66 (1964), 221-231. MR 30 \#2195.

5. G. A. Kamenskii, On the solutions of a linear homogeneous second order differential equation of the unstable type, Trudy Sem. Teor. Differencial. Uravnenirs Otklon. Argumentom Univ. Družby Narodov Patrisa Lumumby 2 (1963), 82-93. (Russian)

6. I. T. Kiguradze, Oscillatory properties of certain ordinary differential equations, Soviet Math. Dokl. 3 (1962), 649-652. (Russian)

7. V. A. Kondrat'ev, Oscillatory proper.ies of solutions of the equation $y^{(n)}+p(x) y=0$, Trudy Moskov. Mat. Obšč. 10 (1961), 419-4j6. MR 25 \# 5239. (Russian)

8. G. Ladas, V. Lakshmikantham and J. S. Papadakis, Oscillations of higher-order retarded differential equations generated by the relarded argument, Delay and Functional Differential Equations and their Applications (K. Schtitt, Ed.), Academic Press, New York, 1972.

9. D. L. Lovelady, Oscillation and even order linear differential equations, Rocky Mountain J. Math. 6 (1976), 299-304.

10. - Oscillation and a clas: of odd order linear differential equa'ions, Hiroshima Math. J. 5 (1975), 371-383.

11. - An asymptotic analysis of an even order linear differential eyuation, Funkcial. Ekvac. (to appear).

12. - An asymptotic analysis of an odd order linear differential equations, Pacific J. Math. 57 (1975), 475-480.

13. - A nonoscillation criterion for a second order delay differential equation (to appear).

14. S. B. Norkin, Differential equations of the second order with retarded arguments, Izdat. "Nauka", Moscow, 1965; English transl., Transl. Math. Monographs, vol. 31, Amer. Math. Soc., Providence, R.I., 1972. MR 33 \#7656.

15. Y. G. Sficas, The effect of the delas on the ascillatory and asymptotic behavior of nth order retarded differential equations, J. Math. Anal. Appl. (to appear).

16. - Retarded actions on oscillations (to appear).

17. Y. G. Sficas and V. A. Staikos, Oscillatory and asymptotic characterizations of the solutions of differential equations with deviating arguments, Proc. London Math. Soc. (to appear).

18. - Oscillations of differential equations with deviating arguments (to appear).

19. C. A. Swanson, Comparison and oscillation theory' of linear differential equations, Academic Press, New York, 1968.

20. P. E. Waltman, $A$ note on an oscillation criterion for an equation with a functional argument, Canad. Math. Bull. 11 (1968), 593-595. MR 38 \#6193.

21. T. Kusano and V. Onose, Nonlinear oscillation of a sublinear delay equation of arbitrary order, Proc. Amer. Math. Soc. 40 (1973), 219-224.

Department of Mathematics, Florida State University, Tallahassez, Florida 32306 